\title{
AVALIAÇÃo PSICOLÓGICA DE CRIANÇAS COM DERMATITE ATÓPICA POR MEIO DO TESTE DAS FÁBULAS DE DÜSS
}

\author{
Maria Rita Polo Gascon \\ Psicóloga da Divisão de Psicologia do Hospital das Clinicas da Universidade de São Paulo - Instituto
}

Central

Mariana Cagnoni Bonfim

Aluna do Curso de Aprimoramento em Psicologia Hospitalar do Instituto Central/Faculdade de

Medicina da Universidade de São Paulo

Tatiane Guimaraes Pedroso

Aluna do Curso de Aprimoramento em Psicologia Hospitalar do Instituto Central/Faculdade de

Medicina da Universidade de São Paulo

Tarcila Rodrigues Campos

Psicóloga da Divisão de Psicologia do Hospital das Clínicas - Instituto Central/Faculdade de

Medicina da Universidade de São Paulo

Glaucia Rosana Guerra Benute

Diretora de Pesquisa da Divisão de Psicologia do Hospital das Clínicas - Instituto Central/Faculdade de Medicina da Universidade de São Paulo

Valéria Aoki

Médica do Ambulatório de Dermatite Atópica da Clinica de Dermatologia do Hospital das Clínicas Instituto Central/Faculdade de Medicina da Universidade de São Paulo

Roberto Takaoka

Médico do Ambulatório de Dermatite Atópica da Clinica de Dermatologia do Hospital das Clínicas Instituto Central/Faculdade de Medicina da Universidade de São Paulo

Raquel Orfali Leão

Médica do Ambulatório de Dermatite Atópica da Clinica de Dermatologia do Hospital das Clínicas Instituto Central/Faculdade de Medicina da Universidade de São Paulo

Mara Cristina Souza De Lúcia
Diretora da Divisão de Psicologia do Hospital das Clínicas - Instituto Central/Faculdade de Medicina
da Universidade de São Paulo

Cyro Festa Neto

Professor Titular da Clínica de Dermatologia do Hospital das Clínicas - Instituto Central/Faculdade de Medicina da Universidade de São Paulo

\section{Resumo}

A Dermatite Atópica (DA) é uma doença inflamatória da pele, de caráter crônico e recorrente, caracterizada por prurido intenso e lesões de pele de distribuição típica. Além do caráter hereditário, a doença pode ser influenciada por fatores ambientais e psicológicos. O objetivo deste estudo foi analisar e refletir sobre o uso do Teste de Fábulas de Düss como um instrumento para compreensão dos aspectos psicodinâmicos de crianças com DA. Foram avaliados 33 pacientes entre 05 e 10 anos, de ambos os sexos com Dermatite Atópica em acompanhamento regular no Ambulatório de Alergia de um Hospital Universitário. Com base nos resultados, foi possível verificar que o teste das Fábulas de Düss se mostra uma técnica que possibilita a expressão dos conflitos emocionais e para a descoberta do funcionamento psíquico destes pacientes. Palavras-chave: dermatite atópica; técnica projetiva; teste das fábulas de Düss. 


\title{
PSYCHOLOGICAL EVALUATION OF CHILDREN ATOPIC DERMATITIS BY DÜSS TEST
}

\begin{abstract}
The Atopic Dermatitis is a skin inflammatory disease, chronic and recurrent, characterized by intense itching and skin lesions with typical distribution. Besides the hereditary character, this disease can be influenced by environmental and psychological factors. The objective of this study was to discuss the use of the Fable of Duss test as a projective and psychodiagnostic instrument for children with AD, in order to understand the psychodinamic aspects. We evaluated 33 patients with atopic dermatitis on regular attendance at the Allergic Clinic of Dermatology Department of University Hospital, between 5 and 10 years of both sexes. Based on the results, it was possible to verify that the Fable of Duss test technique for expression emotional conflicts and discovery of the psychic functioning of these patients.
\end{abstract}

Keywords: atopic dermatitis; projective techniques; Düss test.

\section{EVALUACIÓN PSICOLÓGICA DE NIÑOS CON DERMATITIS ATOPICA POR MEDIO DEL TEST DE LAS FÁBULAS}

\begin{abstract}
Resumen
La Dermatitis Atopica (DA) es una enfermedad inflamatoria de la piel, caracterizada por prurido intenso y lesiones de piel de distribución típica. Además del carácter hereditário, la enfermedad puede estar asociada a factores ambientales y psicológicos. El objetivo de este trabajo ha sido analizar y reflexionar respecto a la utilización Del Test Fábulas de Düss como un instrumento para comprender los aspectos psicodinámicos de niños con DA. Se evaluaron 33 pacientes entre 05 y 10 años, de ambos sexos con dermatitis atópica seguidos de forma regular en la Clínica de Alergia de un Hospital Universitario. Teniendo como base estos resultados, se hizo posible verificar que el test de las Fábulas de Düss se muestra una técnica psicodiagnóstica para la expresión de conflictos emocionales y para el descubrimiento del funcionamiento psíquico de estos pacientes.
\end{abstract}

Palabras clave: dermatitis atópica; técnica proyectiva; fábulas de Düss.

\section{INTRODUÇÃO}

A Dermatite Atópica (DA) é uma doença inflamatória crônica e recorrente da pele, caracterizada por prurido intenso e lesões de distribuição típica. Iniciase geralmente na infância, nos primeiros anos de vida e há uma tendência de involução no decorrer dos anos, persistindo em apenas $20 \%$ dos pacientes após a adolescência (Costa \& Nogueira, 2010; Addor \& Aoki, 2010). Geralmente está associada a outras doenças atópicas como a rinite alérgica e a asma (Cestari, 2010). O diagnóstico é eminentemente clínico baseado na história e manifestações clinicas do paciente e feito através de critérios diagnósticos definidos, ou seja, o paciente deve apresentar prurido, junto com três ou mais dos seguintes achados: história de dermatite flexural, história de alergia respiratória no paciente ou em parente de primeiro grau, pele seca, lesões 
eczematosas ocorrendo antes dos dois anos de idade e eczema presente (Castro et al, 2006). Acredita-se que a inflamação crônica da pele esteja ligada à disfunções da barreira cutânea (Cestari, 2010).

A Dermatite Atópica é influenciada por uma série de fatores, dentre eles os fatores alérgico-irritativos, físicos, infecciosos e psicológicos. Frequentemente é observado que eventos de alto impacto emocional podem causar a exacerbação da doença. A estreita interação da piora da doença com os aspectos emocionais apresentados pelos pacientes explica sua designação de doença psicossomática, reconhecendo-se uma limitação em expressar e nomear sentimentos (Fontes Neto, Weber, Fortes \& Cestari, 2006), sendo a doença uma forma não verbal da expressão de seus conflitos emocionais (McDougall, 2000). Winnicott (1969/1994a) a doença de pele pode ser vista como uma forma de agressão ao meio, pelo fato de ser visível, porém, ao mesmo tempo em que causa repulsa, age como um pedido de cuidado e aproximação.

De acordo com Sampaio e Rivitti (2007) é inegável que os aspectos psicológicos exercem influência nas doenças de pele, podendo assim intensificar ou iniciar tais afecções cutâneas. Tal influência emocional reflete nos sintomas comportamentais das crianças com DA. Um estudo conduzido por Fontes Neto (2005) aponta dificuldades comportamentais e emocionais como ansiedade, depressão, insegurança, teimosia e agressividade. De acordo com a pesquisa, Apontaram também, pouca resiliência dessa população diante do enfrentamento de conflitos.

De acordo com a mesma pesquisa crianças com DA não necessariamente apresentam dificuldade de sociabilização ou de desempenho escolar, porém há um sofrimento diante do medo da não aceitação pelo grupo em que estão inseridos, bem como do preconceito advindo desse. O autor descreve ainda dificuldade de verbalização de sentimentos por estas crianças, sendo expressada por meio de irritação, tensão, agressividade e teimosia.

A doença dermatológica afeta emocionalmente não só o paciente, mas também a sua família que acaba envolvida nas repercussões do processo de adoecer (Costa \& Nogueira, 2010). Por se tratar de uma doença crônica a qual necessita de um tratamento contínuo, a DA possivelmente é a dermatose que requer maior proximidade nas relações entre médico/paciente/família. Por isso, é importante a conscientização da família sobre a doença para uma atuação a fim 
de prevenir ou diminuir riscos ao desenvolvimento biopsicossocial do paciente (Costa \& Nogueira, 2010).

Tendo em vista a importância das relações familiares, e como essas se constituem primariamente na relação estabelecida entre a mãe e o bebê, é necessário atentar-se para as primeiras relações objetais do mesmo (Anzieu, 1989; Spitz, 2004). Dias (2007) aponta estudos psicanalíticos nos quais o adoecimento da pele estaria associado a modelos patológicos de relação objetal internalizada. Essa relação está interrelacionada à constituição do sujeito e a forma como se dá a estruturação do ego. Tal processo ocorre através do contato com o outro, que acontece, em primeiro lugar, pela pele, uma superfície em que o mundo externo e interno imprimem seus conflitos.

A partir dos mesmos estudos embasados em Anzieu, (1989) e McDougall (1978/1989), o adoecimento da pele pode ser pensado como um sintoma relacionado à ruptura de uma união simbiótica, da mãe com a criança, na qual ambas têm uma superfície comum. Através dessa dinâmica da díade mãe-bebê há o processo de investigação do psicólogo, o qual exerce um papel fundamental, pois a ele cabe compreender os aspectos emocionais pertencentes à criança e suas interrrelações com a doença, para auxílio no entendimento biopsicossocial das mesmas.

Em meio a esse cenário, é de fundamental importância realizar um Psicodiagnóstico que contemple a compreensão dos aspectos da personalidade global do paciente ou da dinâmica familiar através do entrelace dos sujeitos envolvidos. Esse processo viabiliza alcançar um entendimento psicodinâmico do caso e possibilitar terapêuticas adequadas (Ocampo, Arzeno, Piccolo e col., 2003).

O Psicodiagnóstico é composto por diferentes etapas e instrumentos, dentre eles, os testes projetivos cuja "percepção é intermediada por elementos singulares à cada sujeito, sendo por estes modelada em maior ou menor grau" (Silva Júnior \& Ferraz, 2001). Dentre as técnicas projetivas, pode-se citar o Teste da Fábula de Düss. De acordo com Cunha e Nunes (1993), o Teste das Fábulas é um método de investigação psicanalítica que envolve determinados complexos, no intuito de obter um olhar clínico mais amplo. O teste também foi avaliado como eficaz para detectar além de um conflito situacional o funcionamento psíquico das crianças. 
As mesmas autoras afirmam que, através do Teste da Fábula de Düss, abre-se uma possibilidade para compreensão da criança em sua linguagem autêntica, isto é, na forma exata como expressa sua realidade, sua fantasia e seu simbolismo. Dessa maneira, o teste é uma via de acesso à identificação do mundo psíquico da criança.

As fábulas são compostas por dez figuras que são apresentadas ao sujeito de forma verbal e pictórica, nas quais o mesmo deve completá-las. Com criança em idade pré-escolar, as fábulas são contadas pelo examinador, ao mesmo tempo em que vão sendo ilustradas com as lâminas correspondentes. A seguir, é realizado um inquérito, que tem a finalidade tanto de estimular a fantasia da criança no desenvolvimento da história, como aprofundar o entendimento psicodinâmico. Esta versão é utilizada preferencialmente com crianças de nível pré-escolar, a partir de 3 anos de idade, podendo ser usada igualmente com escolares, até 8 ou 9 anos. Entretanto, a partir desta faixa etária é preferível a utilização da versão verbal apenas, a não ser no caso de sujeitos muito bloqueados emocionalmente (Cunha, Nunes, 1993). Através dessa técnica, permite-se que a criança manifeste seus conflitos e, muitas vezes, não se aproprie dessa realidade como sua. Dessa forma, o teste das Fábulas de Düss possibilita uma compreensão profunda desses conflitos e, ao mesmo tempo, abarca sua dinâmica familiar (Tardivo, Pinto Júnior \& Santos, 2005).

Tendo em vista a complexidade das relações estabelecidas pela criança com DA e considerando a influência dessas na constituição psíquica bem como as implicações nas terapêuticas realizadas, o objetivo deste estudo foi avaliar e refletir sobre o uso do Teste das Fábulas de Düss como um instrumento projetivo e de compreensão dos aspectos emocionas dessa população.

\section{MÉTODO}

\section{Participantes}

Participaram desta pesquisa 33 pacientes com idade entre 5 e 10 anos, de ambos os sexos, todos com Dermatite Atópica em acompanhamento regular no Ambulatório de Dermatite Atópica de um Hospital Universitário. Foram excluídas crianças que apresentaram os diagnósticos: doenças hereditárias, deficiências mentais ou doenças crônicas. 
O tamanho amostral foi calculado tomando-se por base a quantidade de crianças com Dermatite Atópica em acompanhamento no Ambulatório Dermatológico Especializado de Dermatite Atópica de um Hospital Universitário, em média, 220 pacientes e pela estimativa de dermatite atópica na população de 6 - 7 anos $(8,2 \%)$, acrescidos de $5 \%$ devido à possibilidade de recusa em participar do estudo.

\section{Procedimentos e Instrumentos}

Após a concordância, por escrito, dos responsáveis pela criança , aos itens dispostos no Termo de Consentimento Livre e Esclarecido e assinatura da criança com idade igual ou maior de sete anos do Termo de Assentimento, foram utilizados três instrumentos, a saber, entrevista semi-estruturada, teste de Matrizes Progressivas Coloridas de Raven e teste das Fábulas de Düss.

A entrevista semi-estruturada, elaborada pelos pesquisadores para a presente pesquisa, teve como objetivo coletar dados sociodemográficos, do desenvolvimento e comportamentais das crianças, caracterizando assim a amostra. Foi composta por 24 questões referentes a identificação pessoal e estrutura familiar (pais e criança), renda, posição na ordem de gestação e intercorrências durante o período gestacional, descrição de comportamento da criança quando bebê e na idade em que se encontrava na época da pesquisa, idade em que se apresentaram os sintomas da Dermatite Atópica e se houve hospitalização decorrente da enfermidade.

A fim de avaliar a capacidade cognitiva dos participantes e preencher 0 critério de inclusão ou ausência de deficiência mental, foi utilizado o Teste de Matrizes Progressivas Coloridas de Raven, em sua forma Tabuleiro, como é proposto por Angelini, Custódio, Duarte e Duarte (1999). O instrumento caracteriza-se por um caderno composto por três séries $(A, A B$, e $B)$ cada uma contento 12 itens, um em cada folha. Cada folha constitui-se de desenhos, com um pedaço faltante, possibilitando apenas uma resposta correta. A aplicação desse instrumento se deu conforme as instruções contidas em seu manual e de forma individual.

O Teste das Fábulas de Düss (Cunha \& Nunes, 1993) na versão utilizada nesta pesquisa, compreende uma forma verbal e outra forma pictória. A forma verbal inclui dez pequenas historietas, incompletas, que o sujeito deve completar. A forma pictória é composta por doze lâminas, com ilustrações 
adequadas a cada uma das fábulas, que devem ser apresentadas concomitantemente à forma verbal. As fábulas são as seguintes: 1. Do passarinho; 2. aniversário de casamento; 3. corderinho; 4. enterro ou da viagem; 5 . do medo; 6. do elefante; 7. do objeto fabricado; 8 . do passeio com a mãe ou com o pai; 9. da notícia; 10 . do sonho mau. Há uma lâmina para cada fábula, com exceção da fábula 4, em que deve ser selecionada, entre duas lâminas, a variação que vai utilizar ( a do enterro ou da viagem, nesta pesquisa, utilizamos em todas as aplicações a do enterro) e da fábula 8, em que há lâminas diferentes, dependendo do sexo do sujeito (Cunha, Nunes, 1993).

Análise dos Dados

O método que foi utilizado nesta pesquisa foi quantitativo e qualitativo. Utilizou-se os cálculos de estatística descritiva para obter as informações coletadas na entrevista semiestruturada e para análise qualitativa, utilizou-se o referencial proposto por Tardivo (1998).

Os protocolos das crianças com Dermatite Atópica foram analisados por meio das categorias propostas por Tardivo (1998) comparados com respostas populares contidas no manual de aplicação do Teste da Fábula de Düss (Cunha, Nunes, 1993).

\section{RESULTADOS}

Questionário Sócio-Demográfico: breve caracterização

A amostra da pesquisa foi constituída de $20(60,6 \%)$ crianças do sexo feminino e $13(39,3 \%)$ do sexo masculino, em sua maioria primogênitos $(48,4 \%)$. Segundo relato dos responsáveis, $63,3 \%$ das gestações foram planejadas. Foram referidas intercorrências durante a gestação em 18 (54,4\%) entrevistas, sendo que $6(18,18 \%)$ foram relacionadas diretamente com a gestação, tais como perda de líquido amniótico, ameaça de aborto , sangramento; 5 (15,1\%) depressão; 3 (9\%) falecimento de parentes; $2(6 \%)$ separação conjugal e 2 (6\%) infecção de urina na mãe.

Os sintomas e sinais da Dermatite Atópica se iniciaram em média aos 18,9 meses de vida, 17 (51,5\%) dos pacientes avaliados necessitaram de internação hospitalar devido quadro clínico, apresentando frequência média de internação de 1,5 . 
Quando indagado sobre o comportamento do filho quando bebê, a maioria $(\mathrm{N}=19 ; 57,5 \%)$ o descreveu como tranquilo, enquanto 14 (42,4\%) como agitado. O aleitamento materno foi adotado por $29(87,8 \%)$ das mães entrevistadas, por em média 11,7 meses.

De acordo com os responsáveis, 12 pacientes (36,3\%) apresentavam, no período da pesquisa, comportamento de se isolar no quarto, $11(33,3 \%)$ de roer as unhas, $11(33,3 \%)$ de morder objetos, $2(6 \%)$ de chupar o dedo e $1(3 \%)$ de chupar chupeta, principalmente quando os sintomas da DA estavam presentes.

Em relação ao sono, foi observado, após na análise das respostas dos responsáveis, que 16 crianças $(48,4 \%)$ apresentavam sono tranquilo e 17 $(51,5 \%)$ agitado, sendo os principais comportamentos observados pelos pais enquanto o filho dorme, a coceira em 100\% dos casos, seguido de pesadelos $(\mathrm{N}=9 ; 39,3 \%)$, falar dormindo $(\mathrm{N}=9 ; 27,2 \%)$ e enurese noturna 1 (3\%).

Segundo os pais, o aumento do comportamento de coceira estaria relacionado à ansiedade (27 citações), dificuldade de lidar com a frustração (10 citações), problemas familiares e clima geográfico (2 citações cada). Dois responsáveis entrevistados não relacionaram o aumento do comportamento de coceira com nenhuma circunstância.

Em relação ao comportamento escolar, a maioria $(\mathrm{N}=30 ; 90,9 \%)$ gostava de estudar e apresentava bom rendimento escolar (tendo como base notas escolares) $(\mathrm{N}=31 ; 93,9 \%)$. A interação com os colegas da mesma idade foi descrita como boa por 21 dos responsáveis, $(63,6 \%)$, enquanto $12(36,3 \%)$ relataram dificuldade de contato social com relatos de preconceito devido a enfermidade.

Desenvolvimento Emocional da Criança de acordo com o Teste de Fábulas 


\begin{tabular}{|c|c|c|c|}
\hline Fábula & Categoria & $N(\%)$ & $\begin{array}{c}\text { Respostas } \\
\text { populares } \\
\text { mais } \\
\text { frequentes }\end{array}$ \\
\hline \multirow{5}{*}{$\begin{array}{c}1- \\
\text { Passarinho }\end{array}$} & Autonomia e independência - realista & $19(57,5)$ & $\mathrm{X}$ \\
\hline & Total impotência e morte & $8(24,2)$ & \\
\hline & Relação com a figura materna - independência e atividade & $4(12,1)$ & \\
\hline & Relação com a figura materna - dependência e passividade & $1(3)$ & \\
\hline & Independência e autonomia - onipotente & $1(3)$ & \\
\hline \multirow{3}{*}{$\begin{array}{c}2- \\
\text { Aniversário } \\
\text { de } \\
\text { Casamento }\end{array}$} & $\begin{array}{c}\text { Reação de agressividade e hostilidade da cena primária - } \\
\text { latente }\end{array}$ & $22(66,6)$ & $\mathrm{X}$ \\
\hline & Reação de agressividade diante da cena primária - manifesta & $9(27,2)$ & \\
\hline & Reação de aceitação mais realista da cena primária & $2(6)$ & \\
\hline \multirow{4}{*}{$\begin{array}{l}3 \text { - } \\
\text { Carneirinho }\end{array}$} & Desmame vivido de maneira depressiva & $17(51,5)$ & \\
\hline & Aceitação fraterna & $11(33,3)$ & $\mathrm{X}$ \\
\hline & $\begin{array}{l}\text { Total impotência- impossibilidade de lidar com o desmame ou } \\
\text { morte }\end{array}$ & $4(12,1)$ & \\
\hline & Rivalidade fraterna - agressividade e hostilidades latentes & $1(3)$ & \\
\hline \multirow[t]{9}{*}{4 - Enterro } & Velhice & $8(24,2)$ & $\mathrm{X}$ \\
\hline & Relação com a figura paterna - desejos destrutivos & $6(18,1)$ & \\
\hline & Enfermidade - outras & $6(18,1)$ & \\
\hline & Rejeição à fábula & $6(18,1)$ & \\
\hline & Resposta adequada a realidade & $2(6)$ & \\
\hline & Acidentes & $2(6)$ & \\
\hline & Outros não significativos & $1(3)$ & $x$ \\
\hline & Autodestruição & $1(3)$ & \\
\hline & Provocados por outro & $1(3)$ & \\
\hline \multirow[t]{3}{*}{5 - Medo } & Objetos internos sem definição clara de sexo & $16(48,4)$ & \\
\hline & Objetos reais e externos & $15(45,4)$ & $x$ \\
\hline & Autodestruição & $2(6)$ & \\
\hline \multirow[t]{4}{*}{6 - Elefante } & Superação do complexo de castração & $18(54,5)$ & $x$ \\
\hline & Total impossibilidade de lidar com o complexo de castração & $10(30,3)$ & \\
\hline & $\begin{array}{c}\text { Presença de angústia ligada ao processo de castração - } \\
\text { transformação feita pelo outro }\end{array}$ & $4(12,1)$ & \\
\hline & $\begin{array}{l}\text { Presença de angústia ligada ao processo de castração - } \\
\text { transformações causadas pela própria criança }\end{array}$ & $1(3)$ & \\
\hline \multirow{3}{*}{$\begin{array}{l}7 \text { - Objeto } \\
\text { Fabricado }\end{array}$} & Ausência do caráter possessivo em relação à figura materna & $19(57,5)$ & $x$ \\
\hline & Presença de caráter possessivo em relação á figura materna & $9(27,2)$ & \\
\hline & $\begin{array}{l}\text { Ausência de caráter possessivo em relação à figura materna - } \\
\text { imposição }\end{array}$ & $5(15,1)$ & \\
\hline
\end{tabular}




\begin{tabular}{|c|c|c|c|}
\hline Fábula & Categoria & N (\%) & $\begin{array}{c}\text { Respostas } \\
\text { populares } \\
\text { mais } \\
\text { frequentes }\end{array}$ \\
\hline \multirow{3}{*}{$\begin{array}{c}8 \text { - Passeio } \\
\text { com o pai } \\
\text { ou com a } \\
\text { mãe }\end{array}$} & $\begin{array}{l}\text { Complexo de Édipo vivido de forma angustiante - presença de } \\
\text { culpa depressiva }\end{array}$ & $15(45,4)$ & $\mathrm{x}$ \\
\hline & Complexo de Édipo superado & $13(39,3)$ & \\
\hline & Complexo de Édipo vivido de forma angustiante - raiva e inveja & $1(3)$ & \\
\hline \multirow[t]{4}{*}{9 - Notícia } & Rejeição à fábula & $4(12,1)$ & \\
\hline & Desejos - notícias agradáveis & $23(69,6)$ & $\mathrm{x}$ \\
\hline & Temores - notícias desagradáveis & $8(24,2)$ & \\
\hline & Rejeição à fábula & $2(6)$ & \\
\hline \multirow{4}{*}{$\begin{array}{l}10-\text { Sonho } \\
\text { Mau }\end{array}$} & Relação com circunstâncias difíceis & $18(54,5)$ & $\mathrm{x}$ \\
\hline & Relação com figuras fantásticas & $12(36,3)$ & $\mathrm{x}$ \\
\hline & Pessoas reais & $3(9)$ & \\
\hline & Rejeição à fábula & $3(9)$ & \\
\hline
\end{tabular}

\section{DISCUSSÃO}

A partir das respostas no Teste das Fábulas de Duss, pode-se observar a tendência destes pacientes em reprimir as emoções, dificuldade de expressar raiva, sentimentos de irritabilidade, hostilidade e hipersensibilidade comportamentos já citados por Fontes e Neto (2005) e Fontes Neto, Weber, Fortes \& Cestari (2006), sendo que agressividade presente nos resultados pode se relacionar como a única via de expressão que conseguem dar conta, uma vez que não verbalizam sentimentos, característico de pacientes que sofrem distúrbios psicossomáticos (McDougall, 2000). O comportamento hostil, também observado, pode ser resultado de um medo da não aceitação, por conta de sua doença dermatológica, no grupo em que está inserido (Fontes Neto, 2005).

Tais características refletem nos sintomas emocionais e comportamentais das crianças com DA. Um estudo realizado por Fontes Neto (2005) aponta dificuldades sociais e psíquicas como ansiedade, depressão, insegurança, teimosia e agressividade. Além disso, acredita-se na pouca resiliência dessa população diante do enfrentamento de conflitos, na medida em que eclosões somáticas coincidem, na maioria das vezes, como acontecimentos que ultrapassam a capacidade de tolerância habitual desses pacientes. 
A mesma autora remete a importância dos impulsos agressivos no processo psicossomático. O adoecimento seria o resultado desses impulsos contra o próprio self - já que seria impensável dirigi-lo ao objeto externo - sob forma de culpa. Pode-se observar isto, na fábula 2 (Aniversário de Casamento) onde crianças com dermatite atópica apresentaram mais sentimentos agressivos de maneira latente, ligados à cena primária e na fábula 5 (Medo) onde a resposta mais frequente foi o medo ligado a objetos internos, o que nos sugere que esses objetos possam estar carregados de características negativas, já que, a agressividade está voltada para o próprio individuo.

Em meio a esse contexto, pode-se inferir que a partir desses traços de personalidades somados a uma experiência significativa, como o desmame (observado na fábula 3 - Carneirinho) visto por eles de forma depressiva, essas crianças tenderiam a manifestar comportamentos depressivos e de carência afetiva, podendo também suscitar sentimentos e pensamentos de desprezo e perda do amor materno. É Importante ressaltar que a maioria da amostra é composta por primogênitos e os cuidados maternos foram compartilhados com mais alguém.

Embora a literatura aponte para a dependência como um traço característico dessa população (Fontes Neto, 2005), neste estudo pode-se constatar uma autonomia e independência diante das figuras parentais. Ainda que a criança com DA se expresse demonstrando necessidade de uma dependência parental percebe-se que possuem recursos internos para a busca da independência. Pode-se sugerir, também, uma atitude de proteção em demasia dos pais em relação ao filho com DA tendendo a colocá-los num papel de dependência para além do que realmente precise. Esta atitude de proteção excessiva poderia gerar na criança um prejuízo na elaboração psíquica do complexo de Édipo, vivenciando esta fase com muita culpa depressiva e dificuldade, dados observados na fábula 8 (Passeio com o papai ou a mamãe).

Sendo assim, os resultados do presente estudo, derivados tanto da entrevista com os responsáveis com a mãe, quanto da avaliação feita com a criança por meio da Fábula de Duss somados à articulação com a literatura indicaram uma dificuldade na simbolização e expressão de sentimentos, como raiva e frustração. Outros aspectos observados na avaliação foram: comportamentos de insegurança, desamparo, traços de depressão, sentimento de inferioridade e ressentimento. 
Pode-se constatar, portanto, que a Fábula de Duss é uma importante via de acesso às crianças com DA, complementando um Psicodiagnóstico que abarque uma compreensão mais aprofundada da história de vida, suas variáveis psíquicas, o lugar ocupado nessa família, dentre outros aspectos psíquicos a fim de oferecer uma compreensão integral das crianças com dermatite atópica

\section{REFERÊNCIAS}

Addor, F.A.S \& Aoki, V. (2010). Barreira cutânea na dermatite atópica. An. Bras. Dermatol., 85(2),184-194. Recuperado em 10 setembro, 2010, de http://www.scielo.br/scielo.php?script=sci_arttext\&pid=S0365-

05962010000200009\&Ing $=$ en\&nrm $=$ iso

Angelini L. A; Alves I. C. B; Custódio E. M; Duarte W. F \& Duarte J. L. M. (1999) Manual de matrizes progressivas coloridas de Raven, escala especial. São Paulo: Centro Editor de Testes e Pesquisas em Psicologia.

Anzieu, D. (1989). O Eu-Pele. São Paulo: Casa do Psicólogo.

Castro, A. P. M; Solé, D.; Filho, N. A. R.; Jacob, C. M. A; Rizzo, Ma. C. F. V; Fernandes, M. de F. M. \& Vale, S. O. R. (2006). Guia Prático para o Manejo da Dermatite Atópica - opinião conjunta de especialistas em alergologia da Associação Brasileira de Alergia e Imunopatologia e da Sociedade Brasileira de Pediatria. Rev. Bras. Alerg. Imunopatol., 29(6), 268-282. Recuperado em 26 outubro, 2011, de http://www.asbai.org.br/revistas/Vol296/ART_6_06_Guia_Pratico.pdf

Cestari, S. da C. P. (2010). Dermatite Atópica. In: W. Belda Júnior, N. Di Chiacchio \& P. R. Criado (Ed.). Tratado de Dematologia (Vol 1, Cap 2, pp. 215-238). São Paulo: Atheneu.

Costa, P. M . C \& Nogueira, L. S. C. (2010). Dermatologia Hospitalar. In: W. Belda Júnior, N. Di Chiacchio \& P. R. Criado (Ed.). Tratado de Dematologia (Vol 2, Cap 103, pp. 2216-2215). São Paulo: Atheneu.

Cunha J.A \& Nunes M. L. T. (1993). Teste das Fábulas: Forma verbal e pictória. São Paulo: Centro Editor de Testes e Pesquisas em Psicologia.

Dias, H. Z. J. (2007). Pele e psiquismo, psicossomática e relações objetais: Características relacionais de pacientes portadores de dermatoses. Tese de Doutorado, Pontificia Universidade Católica do Rio Grande do Sul, Porto Alegre, RS, Brasil. 
Fontes Neto, P. T. L, Weber M. B, Fortes S. D, \& Cestari, T. F. (2006). A dermatite atópica na criança: uma visão psicossomática. Rev. Psiquiatr. Rio Gd. Sul, 28(1), 78-82. Recuperado em 14 outubro, 2011, de http://www.scielo.br/scielo.php?script=sci_arttext\&pid=S0101$81082006000100010 \& \operatorname{lng}=$ en\&nrm $=$ iso

Fontes Neto, P. T. L. (2005). Avaliação socio-comportamental de crianças portadoras de dermatite atópica. Dissertação de Mestrado, Universidade Federal do Rio Grande do Sul, Porto Alegre, RS, Brasil.

Ocampo, M. L. S. de, Arzeno, M. E. G.; Piccolo, E. G. e col. (2003). O processo psicodiagnóstico e as técnicas projetivas. (10 $\left.10^{\mathrm{E}} \mathrm{E}\right)$. São Paulo: Martins Fontes.

Mc Dougall (2000). Teatros do corpo: O psicossoma em psicanálise. São Paulo: Martins Fontes.

Sampaio, S.A.P \& Rivitti, E.A. (2007). Dermatologia. (3a Ed). São Paulo: Artes Médicas.

Silva Júnior, N. da \& Ferraz, F. C. (2001). O psicodiagnóstico entre as incompatibilidades de seus instrumentos e as promessas de uma metodologia psicopatológica. Psicol. USP, 12, 179-202. Recuperado em 7 dezembro, 2011, de http://www.scielo.br/scielo.php?script=sci_arttext\&pid=S0103$65642001000100009 \& \operatorname{lng}=$ en\&nrm $=$ iso.

Sptiz, R. (2004). O primeiro ano de vida: Um estudo psicanalítico do desenvolvimento normal e anômalo das relações objetais. (3a Ed). São Paulo: Martins Fontes.

Tardivo, L.S.L.C. (1998) O Teste da apercepção infantil e o teste das Fábulas de Düss: Respostas típicas na população brasileira e aplicações no contexto das técnicas projetivas. São Paulo: Vetor.

Tardivo, L.S.L.C.; Pinto Júnior, A. A. \& Santos, M. R. dos. (2005). Avaliação psicológica de crianças vítimas de violência doméstica por meio do teste das fábulas de Düss. Psic. 6(1), 59-66. Recuperado em 2 outubro, 2011, de http://pepsic.bvsalud.org/scielo.php?script=sci_arttext\&pid=S1676$73142005000100008 \&$ Ing $=$ pt\&nrm $=$ iso. 
Winnicott, D.W. (1994a). Nota adicional sobre transtornos psicossomático. In: C. Winnicott, R. Shepherd, e M.Davis, (Orgs.) Explorações psicanalíticas: D. W. Winnicott (pp.82-90;J.O.A. Abreu, trad.). Porto Alegre: Artmed. (Original publicado em 1964).

Contato: mariaritapolo@yahoo.com.br

Recebido em: 18/07/2012

Revisado em: 24/08/2012

Aceito em: $13 / 11 / 2012$ 